\title{
Copper-Catalyzed Enantioselective Conjugate Addition of Diethylzinc to Acyclic Enones in the Presence of Planar-Chiral Phosphaferrocene-Oxazoline Ligands
}

\author{
Ryo Shintani and Gregory C. Fu* \\ Department of Chemistry \\ Massachusetts Institute of Technology \\ Cambridge, MA 02139
}

\section{Supporting Information}

\section{General}

THF was distilled under nitrogen from sodium benzophenone ketyl prior to use. Toluene and $\mathrm{CH}_{2} \mathrm{Cl}_{2}$ were purified by passage under argon through a neutral alumina column prior to use. $\mathrm{NEt}_{3}$ (EM Science) was distilled from $\mathrm{KOH}$ under argon prior to use. 3,4-Dimethyl-1-phenylphosphole ${ }^{1}$ and 3-cyclohexyl-1-phenyl-2-propen-1-one ${ }^{2}$ were prepared by literature procedures. LiCp* (Strem), ferrocenecarboxaldehyde (Strem), $t$-BuOK (Aldrich), boron trifluoride diethyl etherate (Aldrich), $n$-BuLi solution (Strem), $\mathrm{PhMgCl}$ solution (Aldrich), $\mathrm{FeCl}_{2}$ (Alfa), [Cu(OTf) $]_{2} \cdot \mathrm{C}_{6} \mathrm{H}_{6}$ (Aldrich), $\mathrm{ZnEt}_{2}$ (Aldrich), trifluoroacetic anhydride (Avocado), $\mathrm{MsCl}$ (Aldrich), DMAP (Aldrich), (S)valinol (Fluka), (S)-phenylglycinol (Aldrich or Acros), (R)-phenylglycinol (Avocado), $(1 S, 2 R)-(-)-c i s-1$-amino-2-indanol (Aldrich), acetophenone (Avocado), diisopropylamine (Aldrich), trans-2-octenal (Aldrich), chalcone (Avocado), trans-4-phenyl-3-buten-2-one (Aldrich), 4-methoxychalcone (Aldrich), and 4-chlorochalcone (Avocado) were used as

(1) Breque, A.; Mathey, F.; Savignac, P. Synthesis 1981, 983-985.

(2) Posner, G. H.; O’Dowd, H.; Ploypradith, P.; Cumming, J. N.; Xie, S.; Shapiro, T. A. J. Med. Chem. 1998, 41, 2164-2167. 
received. All the other chemicals and solvents were purchased from either Mallinckrodt or J. T. Baker and used as received.

All air- and moisture-sensitive manipulations were carried out with standard Schlenk techniques under argon or in a glove box under nitrogen. 


\section{Syntheses of Phosphaferrocene-Oxazoline Ligands}

All reactions are unoptimized.

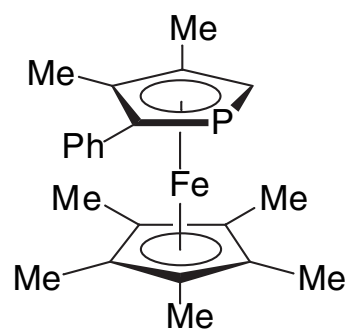

( \pm )-1', $2^{\prime}, 3,3^{\prime}, 4,4^{\prime}, 5^{\prime}-$-Heptamethyl-2-phenylphosphaferrocene (5). A solution of 3,4dimethyl-1-phenylphosphole $(6.65 \mathrm{~g}, 35.3 \mathrm{mmol})$ and $t$-BuOK $(4.76 \mathrm{~g}, 42.4 \mathrm{mmol})$ in THF $(30 \mathrm{~mL})$ was added to a sealed Schlenk tube and stirred for $14 \mathrm{~h}$ at $140{ }^{\circ} \mathrm{C}$ to give a brown solution. Separately, a suspension of $\operatorname{LiCp}^{*}(5.0 \mathrm{~g}, 35 \mathrm{mmol})$ in THF $(200 \mathrm{~mL})$ was added by syringe over $70 \mathrm{~min}$ to a stirred solution of $\mathrm{FeCl}_{2}(4.46 \mathrm{~g}, 35.2 \mathrm{mmol})$ in THF $(75 \mathrm{~mL})$ at $0{ }^{\circ} \mathrm{C}$. After stirring for an additional $10 \mathrm{~min}$ at $0{ }^{\circ} \mathrm{C}$, the brown solution prepared above was added to the solution of $\left[\mathrm{Cp}^{*} \mathrm{FeCl}\right]_{n}$ along with additional THF (15 $\mathrm{mL}$ ). The mixture was stirred for $70 \mathrm{~h}$ at r.t., and then the solvent was removed under vacuum. The residue was filtered through Celite (pentane washings), and the solvent was removed under reduced pressure. The residue was chromatographed on adsorption alumina (pentane) to give $11.11 \mathrm{~g}$ of an orange-red solid (29.4 $\mathrm{mmol}, 84 \%)$.

${ }^{1} \mathrm{H}$ NMR $\left(\mathrm{CD}_{2} \mathrm{Cl}_{2}, 20{ }^{\circ} \mathrm{C}\right): \delta$ 7.41-7.38 (m, 2H), 7.27-7.21 (m, 2H), 7.18-7.11 (m, $\left.1 \mathrm{H}\right)$, $3.40\left(\mathrm{~d},{ }^{2} J_{\mathrm{PH}}=36.0 \mathrm{~Hz}, 1 \mathrm{H}\right), 2.14(\mathrm{~s}, 3 \mathrm{H}), 2.11(\mathrm{~s}, 3 \mathrm{H}), 1.72(\mathrm{~s}, 15 \mathrm{H}) .{ }^{13} \mathrm{C} \mathrm{NMR}\left(\mathrm{CD}_{2} \mathrm{Cl}_{2}, 20\right.$ $\left.{ }^{\circ} \mathrm{C}\right): \delta 141.1\left(\mathrm{~d},{ }^{2} J_{\mathrm{CP}}=17.6 \mathrm{~Hz}\right), 129.6\left(\mathrm{~d},{ }^{3} J_{\mathrm{CP}}=9.1 \mathrm{~Hz}\right), 128.0,125.4,99.9\left(\mathrm{~d},{ }^{1} J_{\mathrm{CP}}=55.1\right.$ $\mathrm{Hz}), 96.7\left(\mathrm{~d},{ }^{2} J_{\mathrm{CP}}=6.7 \mathrm{~Hz}\right), 88.3\left(\mathrm{~d},{ }^{2} J_{\mathrm{CP}}=4.2 \mathrm{~Hz}\right), 82.9,81.8\left(\mathrm{~d},{ }^{1} J_{\mathrm{CP}}=56.9 \mathrm{~Hz}\right), 15.1,13.6$, $10.8\left(\mathrm{~d},{ }^{3} J_{\mathrm{CP}}=0.8 \mathrm{~Hz}\right) \cdot{ }^{31} \mathrm{P}\left\{{ }^{1} \mathrm{H}\right\} \mathrm{NMR}\left(\mathrm{CD}_{2} \mathrm{Cl}_{2}, 2{ }^{\circ} \mathrm{C}\right): \delta-56.4$. M.p. $70-73{ }^{\circ} \mathrm{C}$. FTIR (neat) $3031,2972,2903,1596,1495,1479,1445,1375,1072,1029,833,756,699,668 \mathrm{~cm}^{-1}$. HRMS (EI) calcd for $\mathrm{C}_{22} \mathrm{H}_{27} \mathrm{FeP}\left(\mathrm{M}^{+}\right)$378.1194, found 378.1209. 


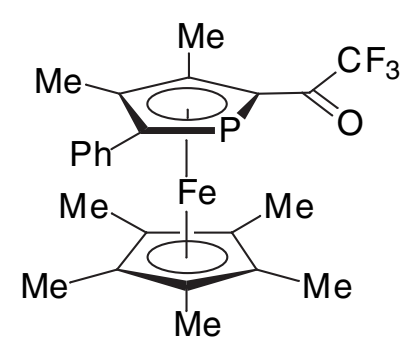

( \pm )-1', $2^{\prime}, 3,3^{\prime}, 4,4^{\prime}, 5^{\prime}$-Heptamethyl-5-phenyl-2-trifluoroacetylphosphaferrocene (6).

Trifluoroacetic anhydride $(554 \mu \mathrm{L}, 3.92 \mathrm{mmol})$ and then boron trifluoride diethyl

etherate ( $497 \mu \mathrm{L}, 3.92 \mathrm{mmol})$ were added by syringe to a stirred solution of $( \pm)-(5)$ (742 $\mathrm{mg}, 1.96 \mathrm{mmol})$ in $\mathrm{CH}_{2} \mathrm{Cl}_{2}(12 \mathrm{~mL})$ at $0{ }^{\circ} \mathrm{C}$. The mixture was stirred for $1.5 \mathrm{~h}$ at r.t. to provide a purple solution, which was chromatographed $\left(\mathrm{Et}_{2} \mathrm{O}\right)$ to give $826 \mathrm{mg}$ of a redbrown solid (1.74 mmol, 89\%).

${ }^{1} \mathrm{H}$ NMR $\left(\mathrm{CD}_{2} \mathrm{Cl}_{2}, 2{ }^{\circ} \mathrm{C}\right): \delta 7.46-7.43(\mathrm{~m}, 2 \mathrm{H}), 7.34-7.23(\mathrm{~m}, 3 \mathrm{H}), 2.38(\mathrm{~s}, 3 \mathrm{H}), 2.22(\mathrm{~s}$, $3 \mathrm{H}), 1.59(\mathrm{~s}, 15 \mathrm{H}) \cdot{ }^{13} \mathrm{C} \mathrm{NMR}\left(\mathrm{CD}_{2} \mathrm{Cl}_{2}, 20{ }^{\circ} \mathrm{C}\right): \delta 189.9\left(\mathrm{qd},{ }^{2} J_{\mathrm{CF}}=33.0 \mathrm{~Hz}\right.$ and ${ }^{2} J_{\mathrm{CP}}=22.8$ $\mathrm{Hz}), 138.1\left(\mathrm{~d},{ }^{2} J_{\mathrm{CP}}=18.7 \mathrm{~Hz}\right), 130.0\left(\mathrm{~d},{ }^{3} J_{\mathrm{CP}}=9.9 \mathrm{~Hz}\right), 128.4,126.8,117.0\left(\mathrm{q},{ }^{1} J_{\mathrm{CF}}=292.8\right.$ $\mathrm{Hz}), 106.3\left(\mathrm{dq},{ }^{1} J_{\mathrm{CP}}=53.6 \mathrm{~Hz}\right.$ and $\left.{ }^{3} J_{\mathrm{CF}}=4.4 \mathrm{~Hz}\right), 99.5\left(\mathrm{~d},{ }^{2} J_{\mathrm{CP}}=5.1 \mathrm{~Hz}\right), 96.9\left(\mathrm{~d},{ }^{2} J_{\mathrm{CP}}=5.7\right.$ $\mathrm{Hz}), 85.3,77.4\left(\mathrm{~d},{ }^{1} J_{\mathrm{CP}}=67.0 \mathrm{~Hz}\right), 13.8,13.8,10.1 .{ }^{19} \mathrm{~F} \mathrm{NMR}\left(\mathrm{CD}_{2} \mathrm{Cl}_{2}, 20{ }^{\circ} \mathrm{C}\right): \delta-72.6\left(\mathrm{~d},{ }^{4} J_{\mathrm{PF}}\right.$ $=62.7 \mathrm{~Hz}) \cdot{ }^{31} \mathrm{P}\left\{{ }^{1} \mathrm{H}\right\} \mathrm{NMR}\left(\mathrm{CD}_{2} \mathrm{Cl}_{2}, 20{ }^{\circ} \mathrm{C}\right): \delta-33.6\left(\mathrm{q},{ }^{4} J_{\mathrm{PF}}=61.7 \mathrm{~Hz}\right)$. M.p. $145-147{ }^{\circ} \mathrm{C}$ (dec). FTIR (neat) 2981, 2909, 1672, 1597, 1481, 1446, 1378, 1303, 1234, 1195, 1168, 1141, 1096, 1028, 927, 758, 732, $701 \mathrm{~cm}^{-1}$. HRMS (EI) calcd for $\mathrm{C}_{24} \mathrm{H}_{26} \mathrm{~F}_{3} \mathrm{FeOP}\left(\mathrm{M}^{+}\right)$474.1017, found 474.1006 .
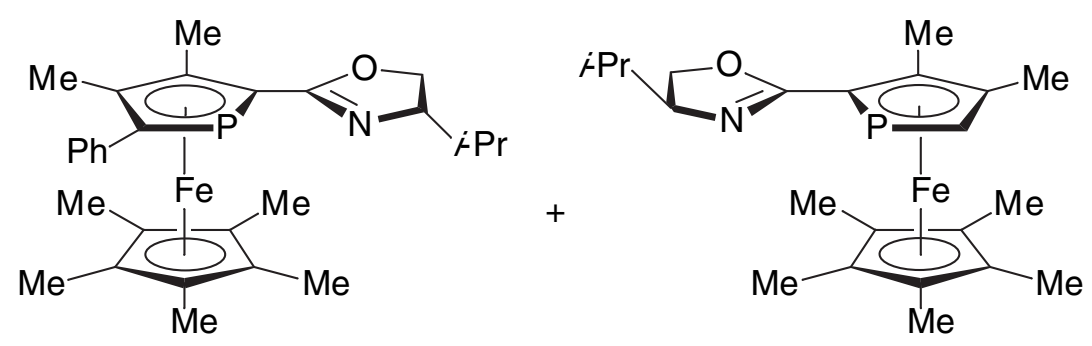

7a and 7a'. $n$-BuLi (1.6 M solution in hexane; $1.97 \mathrm{~mL}, 3.15 \mathrm{mmol}$ ) was added to a stirred solution of $(S)$-valinol $(163 \mathrm{mg}, 1.58 \mathrm{mmol})$ in THF $(7.0 \mathrm{~mL})$ at $0{ }^{\circ} \mathrm{C}$. The reaction mixture was stirred for $2.5 \mathrm{~h}$ at r.t., cooled to $-70{ }^{\circ} \mathrm{C}$, and then a solution of $( \pm)-6$ (250 $\mathrm{mg}, 0.53 \mathrm{mmol})$ in THF $(7.0 \mathrm{~mL})$ was added. After stirring for $20 \mathrm{~h}$ at $60{ }^{\circ} \mathrm{C}$, the reaction mixture was concentrated and then passed through silica gel (washing with $\mathrm{Et}_{2} \mathrm{O}$ ) to 
furnish $207 \mathrm{mg}$ of an orange oil, which was used without further purification.

$\mathrm{MsCl}(63 \mu \mathrm{L}, 0.81 \mathrm{mmol})$ was added to a $-70{ }^{\circ} \mathrm{C}$ mixture of $75.5 \mathrm{mg}$ of this orange oil, DMAP (3.4 mg, $0.028 \mathrm{mmol})$, and $\mathrm{NEt}_{3}(114 \mu \mathrm{L}, 0.82 \mathrm{mmol})$ in $\mathrm{CH}_{2} \mathrm{Cl}_{2}(7.0 \mathrm{~mL})$. The reaction mixture was stirred for $30 \mathrm{~min}$ at $0{ }^{\circ} \mathrm{C}$ and then warmed to r.t. Additional $\mathrm{NEt}_{3}$ ( $284 \mu \mathrm{L}, 2.04 \mathrm{mmol}$ ) was added, and the reaction mixture was stirred for $17 \mathrm{~h}$ at r.t. The solvent was removed under reduced pressure, and the residue was chromatographed $\left(\mathrm{Et}_{2} \mathrm{O} /\right.$ hexane $\left.=1 / 6\right)$. The first fraction $\left(\mathrm{R}_{\mathrm{f}}=0.5\right)$ consisted of $68.6 \mathrm{mg}$ of orange solid $7 \mathbf{a}$ $(0.14 \mathrm{mmol}, 27 \%)$, and the second fraction $\left(\mathrm{R}_{\mathrm{f}}=0.4\right)$ consisted of $52.8 \mathrm{mg}$ of orange solid 7a' (0.11 mmol, 20\%).

7a: ${ }^{1} \mathrm{H}$ NMR $\left(\mathrm{CD}_{2} \mathrm{Cl}_{2}, 20{ }^{\circ} \mathrm{C}\right): \delta$ 7.42-7.38 (m, 2H), 7.29-7.23 (m, 2H), 7.19-7.14 (m, $\left.1 \mathrm{H}\right)$, 4.24-4.15 (m, 1H), 3.91-3.84 (m, 2H), $2.42(\mathrm{~s}, 3 \mathrm{H}), 2.18(\mathrm{~s}, 3 \mathrm{H}), 1.63(\mathrm{~s}, 15 \mathrm{H}), 1.76-1.60(\mathrm{~m}$, $1 \mathrm{H}), 1.01\left(\mathrm{~d},{ }^{3} \mathrm{~J}_{\mathrm{HH}}=6.9 \mathrm{~Hz}, 3 \mathrm{H}\right), 0.88\left(\mathrm{~d},{ }^{3} J_{\mathrm{HH}}=6.9 \mathrm{~Hz}, 3 \mathrm{H}\right) .{ }^{13} \mathrm{C} \mathrm{NMR}\left(\mathrm{CD}_{2} \mathrm{Cl}_{2}, 20{ }^{\circ} \mathrm{C}\right)$ : $\delta 166.4\left(\mathrm{~d},{ }^{2} J_{\mathrm{CP}}=18.1 \mathrm{~Hz}\right), 140.1\left(\mathrm{~d},{ }^{2} J_{\mathrm{CP}}=18.1 \mathrm{~Hz}\right), 130.0\left(\mathrm{~d},{ }^{3} J_{\mathrm{CP}}=9.4 \mathrm{~Hz}\right), 128.3,126.0$, $101.2\left(\mathrm{~d},{ }^{1} J_{\mathrm{CP}}=52.8 \mathrm{~Hz}\right), 95.5\left(\mathrm{~d},{ }^{2} J_{\mathrm{CP}}=5.1 \mathrm{~Hz}\right), 92.7\left(\mathrm{~d},{ }^{2} J_{\mathrm{CP}}=5.2 \mathrm{~Hz}\right), 83.8,79.0\left(\mathrm{~d},{ }^{1} J_{\mathrm{CP}}=\right.$ $54.3 \mathrm{~Hz}), 74.0\left(\mathrm{~d},{ }^{4} J_{\mathrm{CP}}=1.7 \mathrm{~Hz}\right), 69.1,33.8,19.5,18.7,14.1,13.5,10.0\left(\mathrm{~d},{ }^{3} J_{\mathrm{CP}}=0.7 \mathrm{~Hz}\right)$. ${ }^{31} \mathrm{P}\left\{{ }^{1} \mathrm{H}\right\}$ NMR $\left(\mathrm{CD}_{2} \mathrm{Cl}_{2}, 20^{\circ} \mathrm{C}\right): \delta-43.9$. M.p. $95-97^{\circ} \mathrm{C} .[\alpha]^{20}{ }_{\mathrm{D}}+92.7$ (c 0.70, THF). FTIR (neat) 2956, 2903, 1635, 1457, 1377, 1271, 1030, 758, $700 \mathrm{~cm}^{-1}$. HRMS (EI) calcd for $\mathrm{C}_{28} \mathrm{H}_{36} \mathrm{FeNOP}\left(\mathrm{M}^{+}\right)$489.1878, found 489.1866.

7a': ${ }^{1} \mathrm{H}$ NMR $\left(\mathrm{CD}_{2} \mathrm{Cl}_{2}, 20{ }^{\circ} \mathrm{C}\right): \delta$ 7.43-7.39 $(\mathrm{m}, 2 \mathrm{H})$, 7.29-7.23 (m, $\left.2 \mathrm{H}\right), 7.19-7.16(\mathrm{~m}$, 1H), 4.30-4.24 (m, 1H), 3.87-3.72 (m, 2H), $2.41(\mathrm{~s}, 3 \mathrm{H}), 2.19(\mathrm{~s}, 3 \mathrm{H}), 1.64(\mathrm{~s}, 15 \mathrm{H}), 1.75-1.57$ $(\mathrm{m}, 1 \mathrm{H}), 1.12\left(\mathrm{~d},{ }^{3} \mathrm{JHH}_{\mathrm{HH}}=6.6 \mathrm{~Hz}, 3 \mathrm{H}\right), 0.92\left(\mathrm{~d},{ }^{3} \mathrm{~J}_{\mathrm{HH}}=6.9 \mathrm{~Hz}, 3 \mathrm{H}\right) .{ }^{13} \mathrm{C} \mathrm{NMR}\left(\mathrm{CD}_{2} \mathrm{Cl}_{2}, 20{ }^{\circ} \mathrm{C}\right)$ : $\delta 166.7\left(\mathrm{~d},{ }^{2} J_{\mathrm{CP}}=18.2 \mathrm{~Hz}\right), 140.2\left(\mathrm{~d},{ }^{2} J_{\mathrm{CP}}=18.0 \mathrm{~Hz}\right), 129.9\left(\mathrm{~d},{ }^{3} J_{\mathrm{CP}}=9.0 \mathrm{~Hz}\right), 128.3,126.0$, $101.0\left(\mathrm{~d},{ }^{1} J_{\mathrm{CP}}=53.1 \mathrm{~Hz}\right), 95.2\left(\mathrm{~d},{ }^{2} J_{\mathrm{CP}}=4.8 \mathrm{~Hz}\right), 92.5\left(\mathrm{~d},{ }^{2} J_{\mathrm{CP}}=5.4 \mathrm{~Hz}\right), 83.8,79.4\left(\mathrm{~d},{ }^{1} J_{\mathrm{CP}}=\right.$ $54.6 \mathrm{~Hz}), 74.6\left(\mathrm{~d},{ }^{4} J_{\mathrm{CP}}=1.6 \mathrm{~Hz}\right), 70.1,34.9,20.4,19.6,14.2,13.5,10.1\left(\mathrm{~d},{ }^{3} J_{\mathrm{CP}}=0.7 \mathrm{~Hz}\right)$. ${ }^{31} \mathrm{P}\left\{{ }^{1} \mathrm{H}\right\}$ NMR $\left(\mathrm{CD}_{2} \mathrm{Cl}_{2}, 20{ }^{\circ} \mathrm{C}\right): \delta-42.4$. M.p. $106-109^{\circ} \mathrm{C}$. $[\alpha]^{20}{ }_{\mathrm{D}}-155.8$ (c 0.62, THF). FTIR (neat) 2956, 2904, 1631, 1597, 1447, 1377, 1295, 1120, 1030, 1009, 758, $700 \mathrm{~cm}^{-1}$. HRMS (EI) calcd for $\mathrm{C}_{28} \mathrm{H}_{36} \mathrm{FeNOP}\left(\mathrm{M}^{+}\right)$489.1878, found 489.1893. 

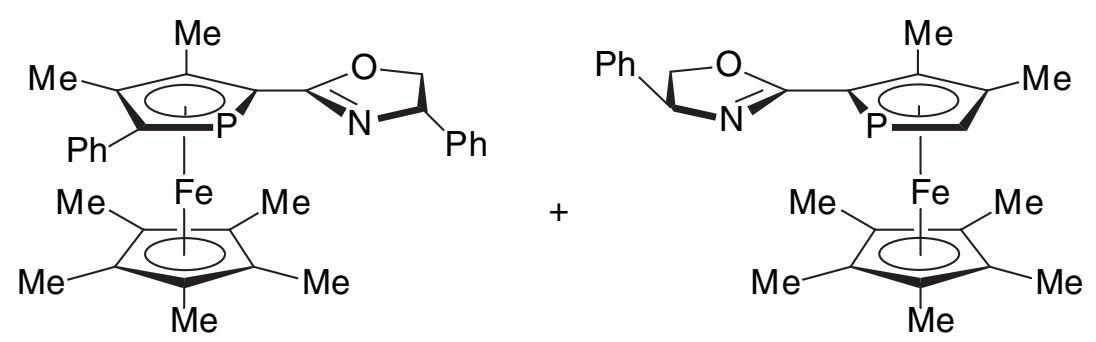

$7 \mathbf{b}$ and $7 b^{\prime}$. The same procedure was followed as for the synthesis of $7 \mathbf{a}$ and $7 a^{\prime}$.

First fraction $\left(\mathrm{R}_{\mathrm{f}}=0.3\right.$ with $\mathrm{Et}_{2} \mathrm{O} /$ hexane $\left.=1 / 7 ; 7 \mathrm{~b}\right)$ : orange solid, $24 \%$. Second fraction $\left(\mathrm{R}_{\mathrm{f}}=0.25\right.$ with $\mathrm{Et}_{2} \mathrm{O} /$ hexane $\left.=1 / 7 ; 7 \mathbf{b}^{\prime}\right)$ : orange solid, $22 \%$.

7b: ${ }^{1} \mathrm{H}$ NMR $\left(\mathrm{CD}_{2} \mathrm{Cl}_{2}, 20{ }^{\circ} \mathrm{C}\right): \delta$ 7.45-7.19 (m, 10H), $5.24\left(\mathrm{dd},{ }^{3} J_{\mathrm{HH}}=9.9\right.$ and $8.3 \mathrm{~Hz}$, $1 \mathrm{H}), 4.59\left(\mathrm{dd},{ }^{3} J_{\mathrm{HH}}=9.9\right.$ and $\left.8.3 \mathrm{~Hz}, 1 \mathrm{H}\right), 3.97\left(\mathrm{t},{ }^{3} J_{\mathrm{HH}}=8.3 \mathrm{~Hz}, 1 \mathrm{H}\right), 2.46(\mathrm{~s}, 3 \mathrm{H}), 2.21(\mathrm{~s}$, $3 \mathrm{H}), 1.67(\mathrm{~s}, 15 \mathrm{H}) .{ }^{13} \mathrm{C}$ NMR $\left(\mathrm{CD}_{2} \mathrm{Cl}_{2}, 20{ }^{\circ} \mathrm{C}\right): \delta 168.5\left(\mathrm{~d},{ }^{2} J_{\mathrm{CP}}=18.1 \mathrm{~Hz}\right), 143.8,139.9(\mathrm{~d}$, $\left.{ }^{2} J_{\mathrm{CP}}=18.0 \mathrm{~Hz}\right), 130.0\left(\mathrm{~d},{ }^{3} J_{\mathrm{CP}}=9.2 \mathrm{~Hz}\right), 129.1,128.3,127.8,127.2,126.1,101.7\left(\mathrm{~d},{ }^{1} J_{\mathrm{CP}}=\right.$ $53.0 \mathrm{~Hz}), 95.6\left(\mathrm{~d},{ }^{2} J_{\mathrm{CP}}=5.0 \mathrm{~Hz}\right), 93.1\left(\mathrm{~d},{ }^{2} J_{\mathrm{CP}}=5.4 \mathrm{~Hz}\right), 84.0,78.1\left(\mathrm{~d},{ }^{1} J_{\mathrm{CP}}=54.9 \mathrm{~Hz}\right), 73.5$, $71.2\left(\mathrm{~d},{ }^{4} J_{\mathrm{CP}}=1.9 \mathrm{~Hz}\right), 14.1,13.7,10.1 .{ }^{31} \mathrm{P}\left\{{ }^{1} \mathrm{H}\right\} \mathrm{NMR}\left(\mathrm{CD}_{2} \mathrm{Cl}_{2}, 20{ }^{\circ} \mathrm{C}\right): \delta-42.7$. M.p. $119-121$ ${ }^{\circ} \mathrm{C} .[\alpha]^{20}{ }_{\mathrm{D}}+149.8$ (c 0.88, THF). FTIR (neat) 2956, 2903, 1627, 1495, 1452, 1377, 1269, 1073, $1020,948,757,699 \mathrm{~cm}^{-1}$. HRMS (EI) calcd for $\mathrm{C}_{31} \mathrm{H}_{34} \mathrm{FeNOP}\left(\mathrm{M}^{+}\right)$523.1722, found 523.1732.

7b': ${ }^{1} \mathrm{H}$ NMR $\left(\mathrm{CD}_{2} \mathrm{Cl}_{2}, 20{ }^{\circ} \mathrm{C}\right): \delta 7.45-7.18(\mathrm{~m}, 10 \mathrm{H}), 5.22\left(\mathrm{t}^{3} J_{\mathrm{HH}}=9.9 \mathrm{~Hz}, 1 \mathrm{H}\right), 4.67$ $\left(\mathrm{dd},{ }^{3} J_{\mathrm{HH}}=9.9\right.$ and $\left.8.3 \mathrm{~Hz}, 1 \mathrm{H}\right), 3.97\left(\mathrm{dd},{ }^{3} J_{\mathrm{HH}}=9.9\right.$ and $\left.8.3 \mathrm{~Hz}, 1 \mathrm{H}\right), 2.44(\mathrm{~s}, 3 \mathrm{H}), 2.21(\mathrm{~s}$, $3 \mathrm{H}), 1.65(\mathrm{~s}, 15 \mathrm{H}) .{ }^{13} \mathrm{C}$ NMR $\left(\mathrm{CD}_{2} \mathrm{Cl}_{2}, 20{ }^{\circ} \mathrm{C}\right): \delta 168.7\left(\mathrm{~d},{ }^{2} J_{\mathrm{CP}}=18.1 \mathrm{~Hz}\right), 143.4,140.0(\mathrm{~d}$, $\left.{ }^{2} J_{\mathrm{CP}}=17.9 \mathrm{~Hz}\right), 130.0\left(\mathrm{~d},{ }^{3} J_{\mathrm{CP}}=9.3 \mathrm{~Hz}\right), 129.0,128.3,127.7,127.2,126.1,101.5\left(\mathrm{~d},{ }^{1} J_{\mathrm{CP}}=\right.$ $53.2 \mathrm{~Hz}), 95.0\left(\mathrm{~d},{ }^{2} J_{\mathrm{CP}}=4.8 \mathrm{~Hz}\right), 92.8\left(\mathrm{~d},{ }^{2} J_{\mathrm{CP}}=5.3 \mathrm{~Hz}\right), 84.0,79.0\left(\mathrm{~d},{ }^{1} J_{\mathrm{CP}}=54.7 \mathrm{~Hz}\right), 73.4$, 71.0, 14.2, 13.8, 10.2. ${ }^{31} \mathrm{P}\left\{{ }^{1} \mathrm{H}\right\}$ NMR $\left(\mathrm{CD}_{2} \mathrm{Cl}_{2}, 20{ }^{\circ} \mathrm{C}\right): \delta-40.9$. M.p. $109-11{ }^{\circ} \mathrm{C} .[\alpha]^{20}{ }_{\mathrm{D}}-241.3$ (c 0.76, THF). FTIR (neat) 2975, 2902, 1624, 1495, 1449, 1377, 1270, 1019, 949, 758, 700 $\mathrm{cm}^{-1}$. HRMS (EI) calcd for $\mathrm{C}_{31} \mathrm{H}_{34} \mathrm{FeNOP}\left(\mathrm{M}^{+}\right)$523.1722, found 523.1705. 

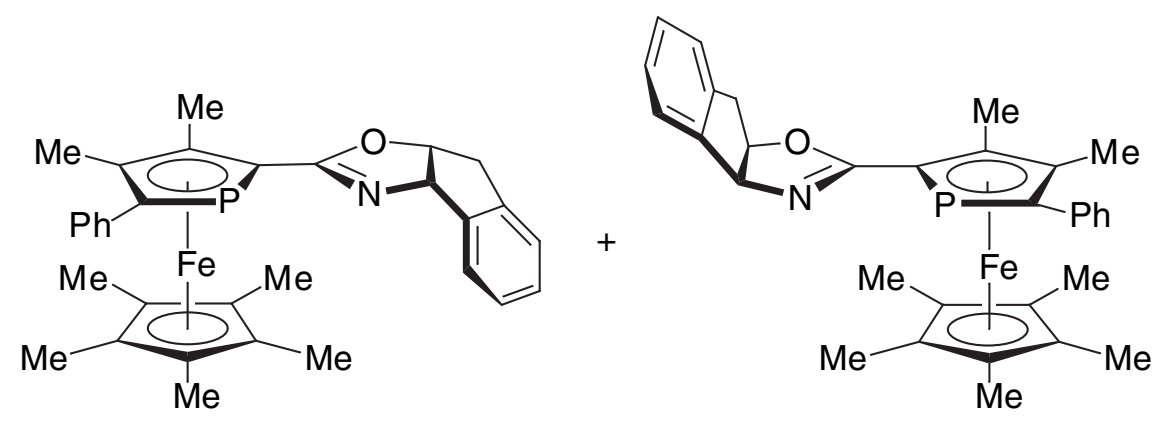

$7 c$ and $7 c^{\prime}$. The same procedure was followed as for the synthesis of $7 \mathbf{a}$ and $7 a^{\prime}$. First fraction $\left(\mathrm{R}_{\mathrm{f}}=0.2\right.$ with $\mathrm{Et}_{2} \mathrm{O} /$ hexane $\left.=1 / 4 ; 7 \mathrm{c}\right)$ : orange solid, $13 \%$. Second fraction $\left(\mathrm{R}_{\mathrm{f}}=0.15\right.$ with $\mathrm{Et}_{2} \mathrm{O} /$ hexane $\left.=1 / 4 ; 7 \mathrm{c}^{\prime}\right)$ : orange solid, $8 \%$.

7c: ${ }^{1} \mathrm{H}$ NMR $\left(\mathrm{CD}_{2} \mathrm{Cl}_{2}, 20{ }^{\circ} \mathrm{C}\right): \delta$ 7.50-7.46 $(\mathrm{m}, 1 \mathrm{H})$, 7.40-7.36 $(\mathrm{m}, 2 \mathrm{H})$, 7.28-7.21 $(\mathrm{m}, 5 \mathrm{H})$, 7.20-7.13 (m, 1H), $5.54\left(\mathrm{~d},{ }^{3} J_{\mathrm{HH}}=8.0 \mathrm{~Hz}, 1 \mathrm{H}\right), 5.26\left(\mathrm{ddd},{ }^{3} J_{\mathrm{HH}}=8.0,6.6\right.$, and $\left.1.7 \mathrm{~Hz}, 1 \mathrm{H}\right)$, $3.44\left(\mathrm{dd},{ }^{3} J_{\mathrm{HH}}=17.9\right.$ and $\left.6.6 \mathrm{~Hz}, 1 \mathrm{H}\right), 3.28\left(\mathrm{dd},{ }^{3} J_{\mathrm{HH}}=17.9\right.$ and $\left.1.4 \mathrm{~Hz}, 1 \mathrm{H}\right), 2.37(\mathrm{~s}, 3 \mathrm{H})$, $2.15(\mathrm{~s}, 3 \mathrm{H}), 1.56(\mathrm{~s}, 15 \mathrm{H}) .{ }^{13} \mathrm{C} \mathrm{NMR}\left(\mathrm{CD}_{2} \mathrm{Cl}_{2}, 20^{\circ} \mathrm{C}\right): \delta 167.8\left(\mathrm{~d},{ }^{2} J_{\mathrm{CP}}=18.0 \mathrm{~Hz}\right), 143.4$, 140.6, $140.1\left(\mathrm{~d},{ }^{2} J_{\mathrm{CP}}=18.0 \mathrm{~Hz}\right), 129.9\left(\mathrm{~d},{ }^{3} J_{\mathrm{CP}}=9.1 \mathrm{~Hz}\right), 128.6,128.2,127.6,126.0,126.0$, 125.6, $101.4\left(\mathrm{~d},{ }^{1} J_{\mathrm{CP}}=53.0 \mathrm{~Hz}\right), 94.9\left(\mathrm{~d},{ }^{2} J_{\mathrm{CP}}=5.0 \mathrm{~Hz}\right), 92.7\left(\mathrm{~d},{ }^{2} J_{\mathrm{CP}}=5.6 \mathrm{~Hz}\right), 83.9,82.1$, $78.6\left(\mathrm{~d},{ }^{1} J_{\mathrm{CP}}=54.3 \mathrm{~Hz}\right), 77.7\left(\mathrm{~d},{ }^{4} J_{\mathrm{CP}}=1.7 \mathrm{~Hz}\right), 40.2,14.1,13.6\left(\mathrm{~d},{ }^{3} J_{\mathrm{CP}}=0.5 \mathrm{~Hz}\right), 9.9\left(\mathrm{~d},{ }^{3} J_{\mathrm{CP}}\right.$ $=0.7 \mathrm{~Hz}) .{ }^{31} \mathrm{P}\left\{{ }^{1} \mathrm{H}\right\} \operatorname{NMR}\left(\mathrm{CD}_{2} \mathrm{Cl}_{2}, 20{ }^{\circ} \mathrm{C}\right): \delta-42.6$. M.p. $144-146{ }^{\circ} \mathrm{C}(\mathrm{dec}) \cdot[\alpha]^{20}{ }_{\mathrm{D}}-2.3(c 0.96$, THF). FTIR (neat) 3025, 2952, 2907, 2854, 1684, 1622, 1496, 1479, 1459, 1427, 1377, 1286, $1264,1197,1167,1073,1026,857,802,757,746,701 \mathrm{~cm}^{-1}$. HRMS (EI) calcd for $\mathrm{C}_{32} \mathrm{H}_{34} \mathrm{FeNOP}\left(\mathrm{M}^{+}\right)$535.1722, found 535.1727.

7c': ${ }^{1} \mathrm{H}$ NMR $\left(\mathrm{CD}_{2} \mathrm{Cl}_{2}, 20{ }^{\circ} \mathrm{C}\right): \delta$ 7.50-7.47 $(\mathrm{m}, 1 \mathrm{H}), 7.42-7.39(\mathrm{~m}, 2 \mathrm{H}), 7.28-7.15(\mathrm{~m}, 6 \mathrm{H})$, $5.56\left(\mathrm{~d},{ }^{3} J_{\mathrm{HH}}=7.4 \mathrm{~Hz}, 1 \mathrm{H}\right), 5.20\left(\mathrm{ddd},{ }^{3} J_{\mathrm{HH}}=7.4,5.2\right.$, and $\left.1.9 \mathrm{~Hz}, 1 \mathrm{H}\right), 3.48-3.35(\mathrm{~m}, 2 \mathrm{H})$, $2.40(\mathrm{~s}, 3 \mathrm{H}), 2.16(\mathrm{~s}, 3 \mathrm{H}), 1.39(\mathrm{~s}, 15 \mathrm{H}) .{ }^{13} \mathrm{C} \mathrm{NMR}\left(\mathrm{CD}_{2} \mathrm{Cl}_{2}, 20{ }^{\circ} \mathrm{C}\right): \delta 168.1\left(\mathrm{~d},{ }^{2} J_{\mathrm{CP}}=18.0\right.$ $\mathrm{Hz}), 143.8,140.7,140.2\left(\mathrm{~d},{ }^{2} J_{\mathrm{CP}}=18.0 \mathrm{~Hz}\right), 129.9\left(\mathrm{~d},{ }^{3} J_{\mathrm{CP}}=9.0 \mathrm{~Hz}\right), 128.6,128.2,127.6$, 126.1, 126.0, 125.5, $101.1\left(\mathrm{~d},{ }^{1} J_{\mathrm{CP}}=52.8 \mathrm{~Hz}\right), 95.4\left(\mathrm{~d},{ }^{2} J_{\mathrm{CP}}=5.1 \mathrm{~Hz}\right), 92.6\left(\mathrm{~d},{ }^{2} J_{\mathrm{CP}}=5.5 \mathrm{~Hz}\right)$, $83.9,82.5,78.3\left(\mathrm{~d},{ }^{1} J_{\mathrm{CP}}=54.6 \mathrm{~Hz}\right), 77.8\left(\mathrm{~d},{ }^{4} J_{\mathrm{CP}}=1.9 \mathrm{~Hz}\right), 39.9,14.1,13.6\left(\mathrm{~d},{ }^{3} J_{\mathrm{CP}}=0.7 \mathrm{~Hz}\right)$, $9.8\left(\mathrm{~d},{ }^{3} J_{\mathrm{CP}}=0.7 \mathrm{~Hz}\right) \cdot{ }^{31} \mathrm{P}\left\{{ }^{1} \mathrm{H}\right\} \mathrm{NMR}\left(\mathrm{CD}_{2} \mathrm{Cl}_{2}, 20{ }^{\circ} \mathrm{C}\right): \delta-43.0$. M.p. $151-153{ }^{\circ} \mathrm{C}(\mathrm{dec}) \cdot[\alpha]^{20}{ }_{\mathrm{D}}$ -183.3 (c 0.95, THF). FTIR (neat) 3052, 2961, 2935, 2903, 1623, 1496, 1478, 1463, 1425, $1377,1310,1270,1239,1196,1164,1120,1095,1053,1023,856,762,749,725,699 \mathrm{~cm}^{-1}$. HRMS (EI) calcd for $\mathrm{C}_{32} \mathrm{H}_{34} \mathrm{FeNOP}\left(\mathrm{M}^{+}\right)$535.1722, found 535.1748. 


\section{Preparation of Enones}

3-Ferrocenyl-1-phenyl-2-propen-1-one. $n$-BuLi (2.6 M solution in hexane; $635 \mu \mathrm{L}$, $1.65 \mathrm{mmol})$ was added to a stirred solution of diisopropylamine $(231 \mu \mathrm{L}, 1.65 \mathrm{mmol})$ in THF $(4 \mathrm{~mL})$ at $-78^{\circ} \mathrm{C}$. The mixture was stirred for $10 \mathrm{~min}$ at $-78^{\circ} \mathrm{C}$ and then warmed to r.t. and stirred for $10 \mathrm{~min}$. The mixture was cooled to $-78{ }^{\circ} \mathrm{C}$, and acetophenone (175 $\mu \mathrm{L}, 1.50 \mathrm{mmol}$ ) was added. This mixture was stirred for $10 \mathrm{~min}$ at $-78{ }^{\circ} \mathrm{C}$, then warmed to r.t. and stirred for $10 \mathrm{~min}$. Then, it was cooled to $-78^{\circ} \mathrm{C}$, and a solution of ferrocenecarboxaldehyde $(321 \mathrm{mg}, 1.50 \mathrm{mmol})$ in THF $(6 \mathrm{~mL})$ was added. The reaction mixture was stirred for $30 \mathrm{~min}$ at $-78^{\circ} \mathrm{C}$, and then warmed to r.t. and stirred for $15 \mathrm{~h}$. The reaction was quenched with sat. aq. $\mathrm{NH}_{4} \mathrm{Cl}$, and the mixture was extracted with $\mathrm{Et}_{2} \mathrm{O}$. The organic layer was dried over $\mathrm{Na}_{2} \mathrm{SO}_{4}$, filtered, and concentrated. The residue was chromatographed under nitrogen (degassed $\mathrm{Et}_{2} \mathrm{O} / \mathrm{CH}_{2} \mathrm{Cl}_{2}=1 / 8$ ), affording $151 \mathrm{mg}$ of the desired product as a purple solid (0.48 mmol, 32\%). The spectroscopic data of this compound were in agreement with previously reported data. ${ }^{3}$

1-Phenyl-2-octen-1-one. trans-2-Octenal $(1.0 \mathrm{~mL}, 6.7 \mathrm{mmol})$ in THF $(6.0 \mathrm{~mL})$ was added to a stirred solution of $\mathrm{PhMgCl}(2.0 \mathrm{M}$ solution in THF; $4.0 \mathrm{~mL}, 8.0 \mathrm{mmol})$ in THF $(6.0 \mathrm{~mL})$ at $0{ }^{\circ} \mathrm{C}$. The mixture was stirred for $3 \mathrm{~h}$ at r.t., then quenched with sat. aq. $\mathrm{NH}_{4} \mathrm{Cl}$ and extracted with $\mathrm{Et}_{2} \mathrm{O}$. The organic layer was dried over $\mathrm{Na}_{2} \mathrm{SO}_{4}$, filtered, and concentrated. The residue was chromatographed $\left(\mathrm{Et}_{2} \mathrm{O} /\right.$ hexane $\left.=1 / 4\right)$ to afford $1.08 \mathrm{~g}$ of a colorless liquid.

This liquid was dissolved in acetone $(10 \mathrm{~mL})$ and cooled to $0^{\circ} \mathrm{C}$. Jones reagent $(1.52$ $\mathrm{M} ; 4.0 \mathrm{~mL}, 6.08 \mathrm{mmol}$ ) was added, and the mixture was stirred for $30 \mathrm{~min}$ at r.t., quenched with isopropanol, and extracted with $\mathrm{Et}_{2} \mathrm{O}$. The organic layer was dried over

$\mathrm{Na}_{2} \mathrm{SO}_{4}$, filtered, and concentrated. The residue was chromatographed $\left(\mathrm{Et}_{2} \mathrm{O} /\right.$ pentane $=$ 1/10) to give $697 \mathrm{mg}$ of colorless liquid (3.45 mmol, 51\%).

${ }^{1} \mathrm{H}$ NMR $\left(\mathrm{CDCl}_{3}, 20{ }^{\circ} \mathrm{C}\right): \delta 7.95-7.91(\mathrm{~m}, 2 \mathrm{H}), 7.58-7.43(\mathrm{~m}, 3 \mathrm{H}), 7.08\left(\mathrm{dt},{ }^{3} J_{\mathrm{HH}}=15.4\right.$

(3) Huang, J.; Huang, Y.-Z. J. Organomet. Chem. 1991, 414, 49-53. 
and $6.9 \mathrm{~Hz}, 1 \mathrm{H}), 6.88\left(\mathrm{dt}^{3}{ }^{3} \mathrm{HH}_{\mathrm{HH}}=15.4\right.$ and $\left.1.1 \mathrm{~Hz}, 1 \mathrm{H}\right), 2.35-2.28(\mathrm{~m}, 2 \mathrm{H}), 1.56-1.48(\mathrm{~m}$, 2H), 1.38-1.31 (m, 4H), $0.91\left(\mathrm{t}^{3} \mathrm{~J}_{\mathrm{HH}}=6.9 \mathrm{~Hz}, 3 \mathrm{H}\right) .{ }^{13} \mathrm{C} \mathrm{NMR}\left(\mathrm{CDCl}_{3}, 20{ }^{\circ} \mathrm{C}\right): \delta 191.2,150.4$, 138.2, 132.8, 128.7, 128.7, 126.0, 33.0, 31.6, 28.0, 22.6, 14.2. FTIR (neat) 3059, 2930, 2858, $1670,1623,1579,1448,1348,1282,1226,1180,1014,988,771,694,662 \mathrm{~cm}^{-1}$. HRMS (EI) calcd for $\mathrm{C}_{14} \mathrm{H}_{18} \mathrm{O}\left(\mathrm{M}^{+}\right)$202.1352, found 202.1347. 


\section{Catalytic Enantioselective Conjugate Additions}

General procedure (Table 1). A mixture of $[\mathrm{Cu}(\mathrm{OTf})]_{2} \cdot \mathrm{C}_{6} \mathrm{H}_{6}(1.9 \mathrm{mg}, 3.8 \mu \mathrm{mol})$ and the ligand $(9.0 \mu \mathrm{mol})$ in toluene $(1.3 \mathrm{~mL})$ was stirred for $1 \mathrm{~h}$ at r.t. Then, the reaction mixture was cooled to $-70{ }^{\circ} \mathrm{C}$, and solutions of the enone $(0.151 \mathrm{mmol})$ in toluene $(0.6$ $\mathrm{mL})$ and $\mathrm{ZnEt}_{2}(23.2 \mu \mathrm{L}, 0.226 \mathrm{mmol})$ in toluene $(0.6 \mathrm{~mL})$ were added. The reaction mixture was stirred for $24 \mathrm{~h}$ at $0{ }^{\circ} \mathrm{C}$, and then it was quenched with $1 \mathrm{~N} \mathrm{HCl}$ and extracted $\left(\mathrm{Et}_{2} \mathrm{O}\right)$. The organic extracts were dried over $\mathrm{Na}_{2} \mathrm{SO}_{4}$, filtered, and concentrated. The residue was chromatographed on silica gel or adsorption alumina $\left(\mathrm{Et}_{2} \mathrm{O} /\right.$ pentane mixtures), thereby furnishing the desired product of conjugate addition.

(S)-(+)-1,3-Diphenyl-1-pentanone (entry 1). Ligand 7c was used. Colorless oil; 82\% yield, $86 \%$ ee. $[\alpha]^{20}{ }_{D}+7.3$ (c 1.52, ethanol).

${ }^{1} \mathrm{H}$ NMR $\left(\mathrm{CDCl}_{3}, 20{ }^{\circ} \mathrm{C}\right): \delta$ 7.91-7.88 (m, 2H), 7.55-7.39 (m, 3H), 7.31-7.14 (m, 5H), 3.31-3.21 (m, 3H), 1.83-1.61 (m, 2H), $0.80\left(\mathrm{t},{ }^{3} \mathrm{~J}_{\mathrm{HH}}=7.3 \mathrm{~Hz}, 3 \mathrm{H}\right) .{ }^{13} \mathrm{C} \mathrm{NMR}\left(\mathrm{CDCl}_{3}, 20{ }^{\circ} \mathrm{C}\right)$ : $\delta 199.4,144.9,137.4,133.1,128.7,128.6,128.3,127.8,126.5,45.8,43.2,29.4,12.3$.

The absolute configuration of the product was determined by comparison of the optical rotation with that reported in the reported literature. ${ }^{4}$ The ee was determined by chiral HPLC: Daicel Chiralcel OD column with hexanes:isopropanol = 99.75:0.25, flow $=1 \mathrm{~mL} / \mathrm{min}$. Retention time: $16.6 \mathrm{~min}$ [(S)-enantiomer], $21.5 \mathrm{~min}$ [(R)-enantiomer].

(S)-(+)-3-(4-Methoxyphenyl)-1-phenyl-1-pentanone (entry 2). Ligand 7c was used. Colorless oil; $78 \%$ yield, $90 \%$ ee. $[\alpha]^{20}{ }_{D}+15.2$ (c 1.67, ethanol).

${ }^{1} \mathrm{H} \mathrm{NMR}\left(\mathrm{CDCl}_{3}, 20{ }^{\circ} \mathrm{C}\right): \delta$ 7.92-7.88 (m, 2H), 7.55-7.49 (m, 1H), 7.45-7.39 (m, 2H), 7.16-7.11 (m, 2H), 6.85-6.80 (m, 2H), $3.76(\mathrm{~s}, 3 \mathrm{H}), 3.27-3.15(\mathrm{~m}, 3 \mathrm{H}), 1.80-1.56(\mathrm{~m}, 2 \mathrm{H})$,

$0.80\left(\mathrm{t},{ }^{3} \mathrm{~J}_{\mathrm{HH}}=7.3 \mathrm{~Hz}, 3 \mathrm{H}\right) .{ }^{13} \mathrm{C} \mathrm{NMR}\left(\mathrm{CDCl}_{3}, 20{ }^{\circ} \mathrm{C}\right): \delta 199.6,158.1,137.4,136.8,133.1$, $128.7,128.7,128.2,113.9,55.3,46.0,42.4,29.5,12.3$.

The absolute configuration of the product was assigned by analogy with entry 1 . The ee was determined by chiral HPLC: Daicel Chiralcel OD column with

(4) Brienne, M.-J.; Ouannes, C.; Jacques, J. Bull. Soc. Chim. Fr. 1967, 32, 613-623. 
hexanes:isopropanol $=99.4: 0.6$, flow $=1 \mathrm{~mL} / \mathrm{min}$. Retention time: $10.4 \mathrm{~min}[(R)-$ enantiomer], $11.4 \mathrm{~min}$ [(S)-enantiomer].

(S)-(+)-3-(4-Chlorophenyl)-1-phenyl-1-pentanone (entry 3). Ligand 7b was used. Colorless oil; $87 \%$ yield, $80 \%$ ee. $[\alpha]^{20}{ }_{D}+2.0$ (c 2.00, ethanol).

${ }^{1} \mathrm{H} \mathrm{NMR}\left(\mathrm{CDCl}_{3}, 20^{\circ} \mathrm{C}\right): \delta 7.91-7.87(\mathrm{~m}, 2 \mathrm{H}), 7.56-7.40(\mathrm{~m}, 3 \mathrm{H}), 7.27-7.13(\mathrm{~m}, 4 \mathrm{H})$, 3.26-3.21 (m, 3H), 1.82-1.57 (m, 2H), $0.80\left(\mathrm{t},{ }^{3} \mathrm{~J}_{\mathrm{HH}}=7.3 \mathrm{~Hz}, 3 \mathrm{H}\right) .{ }^{13} \mathrm{C} \mathrm{NMR}\left(\mathrm{CDCl}_{3}, 20{ }^{\circ} \mathrm{C}\right)$ : $\delta$ 199.0, 143.3, 137.3, 133.2, 132.0, 129.2, 128.8, 128.7, 128.2, 45.6, 42.5, 29.4, 12.2 .

The absolute configuration of the product was assigned by analogy with entry 1 . The ee was determined by chiral HPLC: Daicel Chiralcel OD column with hexanes:isopropanol $=99.95: 0.05$, flow $=1 \mathrm{~mL} / \mathrm{min}$. Retention time: $40.3 \mathrm{~min}[(S)$ enantiomer], $45.1 \mathrm{~min}[(R)$-enantiomer].

(-)-3-Ferrocenyl-1-phenyl-1-pentanone (entry 4). Ligand $7 \mathrm{~b}$ was used. Yellow oil; $88 \%$ yield, $92 \%$ ee. $[\alpha]^{20}{ }_{D}-68.7$ (c 1.80, ethanol).

${ }^{1} \mathrm{H}$ NMR $\left(\mathrm{CDCl}_{3}, 20{ }^{\circ} \mathrm{C}\right): \delta 8.01-7.97(\mathrm{~m}, 2 \mathrm{H}), 7.60-7.45(\mathrm{~m}, 3 \mathrm{H}), 4.10(\mathrm{~s}, 5 \mathrm{H}), 4.09-4.02$ $(\mathrm{m}, 4 \mathrm{H}), 3.32\left(\mathrm{dd},{ }^{3} J_{\mathrm{HH}}=16.5\right.$ and $\left.5.5 \mathrm{~Hz}, 1 \mathrm{H}\right), 3.24\left(\mathrm{dd},{ }^{3} J_{\mathrm{HH}}=16.2\right.$ and $\left.7.4 \mathrm{~Hz}, 1 \mathrm{H}\right), 3.18-$ $3.12(\mathrm{~m}, 1 \mathrm{H}), 1.64-1.54(\mathrm{~m}, 2 \mathrm{H}), 0.87\left(\mathrm{t},{ }^{3} \mathrm{~J}_{\mathrm{HH}}=7.5 \mathrm{~Hz}, 3 \mathrm{H}\right) .{ }^{13} \mathrm{C} \mathrm{NMR}\left(\mathrm{CDCl}_{3}, 20{ }^{\circ} \mathrm{C}\right)$ : $\delta$ 200.0, 137.6, 133.2, 128.8, 128.3, 94.2, 68.6, 67.8, 67.3, 67.2, 66.7, 44.4, 35.7, 29.4, 11.9. FTIR (neat) 3091, 2962, 2931, 2875, 1684, 1597, 1580, 1449, 1357, 1276, 1211, 1106, 1002, $819,753,690 \mathrm{~cm}^{-1}$. HRMS (EI) calcd for $\mathrm{C}_{21} \mathrm{H}_{22} \mathrm{FeO}\left(\mathrm{M}^{+}\right)$346.1015, found 346.1002 .

The absolute configuration of the product was not determined. The ee was determined by chiral HPLC: Daicel Chiralcel OD column with hexanes:isopropanol = 99:1, flow $=1 \mathrm{~mL} / \mathrm{min}$. Retention time: $8.4 \mathrm{~min}, 9.2 \mathrm{~min}$.

(S)-(+)-3-Cyclohexyl-1-phenyl-1-pentanone (entry 5). Ligand 7c' was used. Colorless oil; $68 \%$ yield, $84 \%$ ee. $[\alpha]^{20}{ }_{D}+0.9$ (c 1.51, ethanol).

${ }^{1} \mathrm{H}$ NMR $\left(\mathrm{CDCl}_{3}, 20{ }^{\circ} \mathrm{C}\right): \delta$ 7.97-7.95 (m, 2H), 7.58-7.43 (m, 3H), $2.98\left(\mathrm{dd},{ }^{3} J_{\mathrm{HH}}=16.2\right.$ and $5.5 \mathrm{~Hz}, 1 \mathrm{H}), 2.77\left(\mathrm{dd},{ }^{3} J_{\mathrm{HH}}=16.2\right.$ and $\left.7.4 \mathrm{~Hz}, 1 \mathrm{H}\right), 1.99-1.93(\mathrm{~m}, 1 \mathrm{H}), 1.76-1.62(\mathrm{~m}$, $5 \mathrm{H}), 1.47-1.00(\mathrm{~m}, 8 \mathrm{H}), 0.87\left(\mathrm{t},{ }^{3} \mathrm{~J}_{\mathrm{HH}}=7.4 \mathrm{~Hz}, 3 \mathrm{H}\right) .{ }^{13} \mathrm{C} \mathrm{NMR}\left(\mathrm{CDCl}_{3}, 20{ }^{\circ} \mathrm{C}\right): \delta 201.3,137.7$, 133.0, 128.7, 128.3, 41.3, 40.4, 40.4, 30.5, 29.5, 27.0, 27.0, 27.0, 24.2, 12.1 .

The absolute configuration of the product was determined by comparison of the 
optical rotation with that reported in the reported literature. ${ }^{4}$ The ee was determined by chiral HPLC: Regis (R,R) WHELK-O 2 column with hexanes:isopropanol = 99.75:0.25, flow $=1 \mathrm{~mL} / \mathrm{min}$. Retention time: $21.9 \mathrm{~min}[(R)$-enantiomer], $24.9 \mathrm{~min}[(S)-$ enantiomer].

(+)-3-Ethyl-1-phenyl-1-octanone (entry 6). Ligand 7b was used. Colorless liquid; $79 \%$ yield, $62 \%$ ee. $[\alpha]^{20}{ }_{D}+1.7$ (c 1.60, ethanol).

${ }^{1} \mathrm{H} \mathrm{NMR}\left(\mathrm{CDCl}_{3}, 20{ }^{\circ} \mathrm{C}\right): \delta$ 7.97-7.94 (m, 2H), 7.58-7.43 (m, 3H), $2.87\left(\mathrm{~d},{ }^{3} J_{\mathrm{HH}}=6.9 \mathrm{~Hz}\right.$, $2 \mathrm{H}), 2.07-2.03(\mathrm{~m}, 1 \mathrm{H}), 1.46-1.19(\mathrm{~m}, 10 \mathrm{H}), 0.91-0.85(\mathrm{~m}, 6 \mathrm{H}) .{ }^{13} \mathrm{C} \mathrm{NMR}\left(\mathrm{CDCl}_{3}, 20{ }^{\circ} \mathrm{C}\right)$ : $\delta$ 201.0, 137.7, 133.0, 128.7, 128.3, 43.3, 35.9, 33.7, 32.3, 26.7, 26.6, 22.9, 14.3, 11.1. FTIR (neat) 2959, 2928, 2858, 1684, 1598, 1581, 1449, 1377, 1281, 1213, 1180, 1126, 750, $691 \mathrm{~cm}^{-1}$. HRMS (EI) calcd for $\mathrm{C}_{16} \mathrm{H}_{24} \mathrm{O}\left(\mathrm{M}^{+}\right)$232.1822, found 232.1819.

The absolute configuration of the product was not determined. The ee was determined by chiral HPLC: Daicel Chiralcel OD column with hexanes:isopropanol = 99.95:0.05, flow $=1 \mathrm{~mL} / \mathrm{min}$. Retention time: $13.1 \mathrm{~min}, 13.8 \mathrm{~min}$.

(S)-(+)-4-Phenyl-2-hexanone (entry 7). Ligand $7 \mathbf{b}$ was used. Pale-yellow oil; 82\% yield, $81 \%$ ee. $[\alpha]^{20}{ }_{D}+27.0$ (c 1.32, ethanol).

${ }^{1} \mathrm{H}$ NMR $\left(\mathrm{CDCl}_{3}, 20{ }^{\circ} \mathrm{C}\right): \delta$ 7.32-7.26 (m, 2H), 7.22-7.15 (m, 3H), 3.06-2.98 $(\mathrm{m}, 1 \mathrm{H}), 2.73$ $\left(\mathrm{d},{ }^{3} J_{\mathrm{HH}}=7.4 \mathrm{~Hz}, 2 \mathrm{H}\right), 2.02(\mathrm{~s}, 3 \mathrm{H}), 1.73-1.51(\mathrm{~m}, 2 \mathrm{H}), 0.78\left(\mathrm{t},{ }^{3} J_{\mathrm{HH}}=7.3 \mathrm{~Hz}, 3 \mathrm{H}\right) .{ }^{13} \mathrm{C} \mathrm{NMR}$ $\left(\mathrm{CDCl}_{3}, 20^{\circ} \mathrm{C}\right): \delta 208.3,144.5,128.6,127.7,126.5,50.8,43.2,30.9,29.6,12.2$.

The absolute configuration of the product was determined by comparison of the optical rotation with that reported in the reported literature. ${ }^{4}$ The ee was determined by chiral HPLC: Daicel Chiralcel OD column with hexanes:isopropanol = 99:1, flow $=1$ $\mathrm{mL} / \mathrm{min}$. Retention time: $8.5 \mathrm{~min}[(S)$-enantiomer], $9.4 \mathrm{~min}$ [(R)-enantiomer]. 